\title{
Microscale spectroscopic thermal imaging of $n$-alkanes
}

\author{
by M. Ryu*, M. Romano**, J.C. Batsale**, C. Pradere**, J. Morikawa*,*** \\ *Tokyo Institute of Technology, Dept. of Materials Science and Engineering, School of Materials and Chemical \\ Technology, Tokyo 152-8550, Japan, ryu.m.ab@m.titech.ac.jp, morikawa.j.aa@m.titech.ac.jp. \\ ** I2M, Dpt.TREFLE, UMR CNRS 5295 -ENSAM, Esplanade des Arts et Métiers, 33405 Talence, France, \\ c.pradere@i2m.u-bordeaux1.fr, romano@enscpb.fr, a.sommier@2m.u-bordeaux1.fr, jc.batsale@2m.u-bordeaux1.fr. \\ ${ }^{\star \star *}$ Center for Materials research by Information Integration $\left(\mathrm{CMI}^{2}\right)$, National Institute for Materials Science (NIMS) \\ 1-2-1 Sengen, Tsukuba, Ibaraki 305-0047, JAPAN,
}

\begin{abstract}
Based on the recent development of our team on simultaneous measurement of thermal and spectral IR images, in this paper, the study of phase transition of molecular organic crystals, $n$-Tetraosane $\left(\mathrm{C}_{24} \mathrm{H}_{50}\right)$, as a typical $n$-alkane will be presented. The goal is to demonstrate the high potentiality of the device in the study of couple phenomena.
\end{abstract}

\section{Introduction}

The observation of spectrum distribution in IR range contributes much to the investigation of chemical distribution in the composite material or chemical reaction system [1-5]. There are mainly two ways to measure spectrum distribution. In one method, the focused IR incident light measures the spectrum at limited area and output IR signal is detected by single sensor, then by scanning the spot of measurement, the in-plane spectroscopic information can be obtained (mapping). In another method, the wide spot of the IR light is homogeneously introduced into sample and output light is measured by the array sensor (imaging). The former method is advantageous to the static state measurement and the latter method is to the observation of the dynamic change of system.

The phase change of organic material is the dynamic spectrum change associated with the inflow and outflow of heat. Especially in the cooling, the organic material forms characteristic structure and releases the latent heat. The relation between in-plane evolutions of the organic crystal structure and in-plane latent heat propagation has been investigated [16-18]. Heat propagation in microscale has been observed by the achromatic optical lens systems designed for the micro-scale thermography. Among them, the microscale structure evolution in the organic material crystallization has been widely researched with these methodologies [6-10]. At the same time, IR spectroscopy investigations on the different state of the organic materials have been also made [11-13]. Some specific absorption in organic materials are sensitive to the crystal structure and such bands are used for characterization of the organic molecular crystal. To visualize these two $2 \mathrm{D}$ phenomena simultaneously, the IR imaging and the IR thermography are combined, and the system was applied for the organic material phase change.

Recently, a novel system [14-15] developed by our team, that combined two imaging techniques enabled simultaneous measurements of IR spectroscopic and thermal imaging at multiscale of the time and space. The thermal and the spectroscopic images are alternatively recorded with the synchronized signal from a chopper that attains the high simultaneity in the measurement. Previous measurement [15] performed in steady state demonstrates the ability of the set-up to record concentration and temperature fields simultaneously during phase change of $n$-Eicosane $\left(\mathrm{C}_{20} \mathrm{H}_{42}\right)$ in steady state with a $5 \mathrm{~ms}$ time interval and with a spatial resolution of $4 \mu \mathrm{m}$.

In this paper, the system is applied to study dynamic phase change of $n$-tetracosane $\mathrm{C}_{24} \mathrm{H}_{50}$ at higher frequency $(200 \mathrm{~Hz})$ acquisition in order to demonstrae the high performance of this analytical tool as a new measuring device for the simultaneous thermal and chemical structural analysis of materials at microscale.

To illustrate that, the Fast Infrared Imaging Spectroscopy Technique (FIIST) to realize highly dynamics studies (25000 images at $200 \mathrm{~Hz}$ ) of phase change is demonstrated in the liquid-rotator, and the rotator-solid phase transition of $n$-Tetracosane $\left(\mathrm{C}_{24} \mathrm{H}_{50}\right)$.

\section{Methods and material}

\subsection{Experimental setup}

The multispectral imaging instrument developed in this study is schematized in Figure 1. The instrument is composed of a ceramic heater, a chopper, a grating monochromator to select the excitation wavelength (CT-10, JASCO Corporation), a condenser module with a parabolic mirror, a thin hot stage (S84, ST-Japan) and an IR camera (Phoenix, Indigo) whose detector size is $30 \mu \mathrm{m} \times 30 \mu \mathrm{m}$ and spectral range is from $3.0 \mu \mathrm{m}$ to $5.0 \mu \mathrm{m}$. 
The infrared light emitted by the ceramic lamp (Fig. 1.A.) first passes through the chopper which is able to switch to <on $>$ and $<$ off $>$ state of the irradiation light. The chopper frequency was controlled by DC power supply. The frequency range of the chopper goes from $17 \mathrm{~Hz}$ to $1 \mathrm{KHz}$ with frequency instability of $0.1 \mathrm{~Hz}$ at $100 \mathrm{~Hz}$. The frequency of the chopper was monitored with a photo diode as TTL signal (Transistor-Transistor Logic signal). After the chopper, the optical band pass filter which extracts the light between $2.5 \mu \mathrm{m}$ and $5.5 \mu \mathrm{m}(80 \%$ transmittance) is inserted as the order sorting filter to avoid high order diffraction of the monochromator (Fig. $1 \mathrm{~B}$ ).

A blazed grating with 300 grooves was used to scan over a broad infrared band ranging from 2.76 to $5.24 \mu \mathrm{m}$ with $0.1 \mathrm{~nm}$ steps in this application. The wavelength resolution of the system was controlled by the 2 slits in the monochromator (Figure 1.B.). The opening width of both slits can be changed from $0.1 \mathrm{~mm}$ to $4 \mathrm{~mm}$. The opening width of 2 slits is adjusted with the optimal intensity and wavelength resolution for the measurement. The opening width dependency of the wavelength resolution was calibrated with a typical narrow band pass filter (NB-3555-041, Spectrogon) having a well-defined transmission area. To obtain the relationship between the opening width of the slits and the wavelength resolution, the spectrum of the filter with several wavelength resolutions are calculated, then compared with the experimental data.

The beam emitted from the slit (Fig. 1.B.) is monochromatic and condensed with a parabolic mirror of $1 / 3$ reduction rate (Fig. 1.C.). The IR camera is an $\mathrm{InSb}(3$ to $5 \mu \mathrm{m})$ focal plane array (FPA) composed of $320 \times 256$ pixels and pitch size of $30 \mu \mathrm{m} \times 30 \mu \mathrm{m}$ with $\times 7$ magnification objective lens, the resulting spatial resolution is $4 \mu \mathrm{m}$ (Fig. 1.D.). The maximum frequency acquisition of the camera is $200 \mathrm{~Hz}$ in snapshot mode. The spectral measurements are done in transmission mode, so the camera is placed in front of the sample and the incident light irradiates from the backside of the sample, which is deposited in a microscopic heating stage closed by two $\mathrm{CaF}_{2}$ windows at the top and bottom. Inside the closed hot stage, the temperature is controlled by a PID regulator $\left( \pm 0.1^{\circ} \mathrm{C}\right.$ uncertainty). The temperature is controlled by the water-cooled system in the temperature range going from 10 to $500^{\circ} \mathrm{C}$ and maximum rate of $20^{\circ} \mathrm{C} / \mathrm{min}$.

The thermal and the spectroscopic images are alternatively recorded by the synchronization of the chopper signal and the input trigger of the camera. This allows us to attain high simultaneity in the measurement. The simultaneous acquisition of thermal and spectroscopic images was performed at $200 \mathrm{~Hz}$ alternating acquisition of two kinds of images. They are processing with the two images technique [14], and converted to both spectroscopic and thermal information.

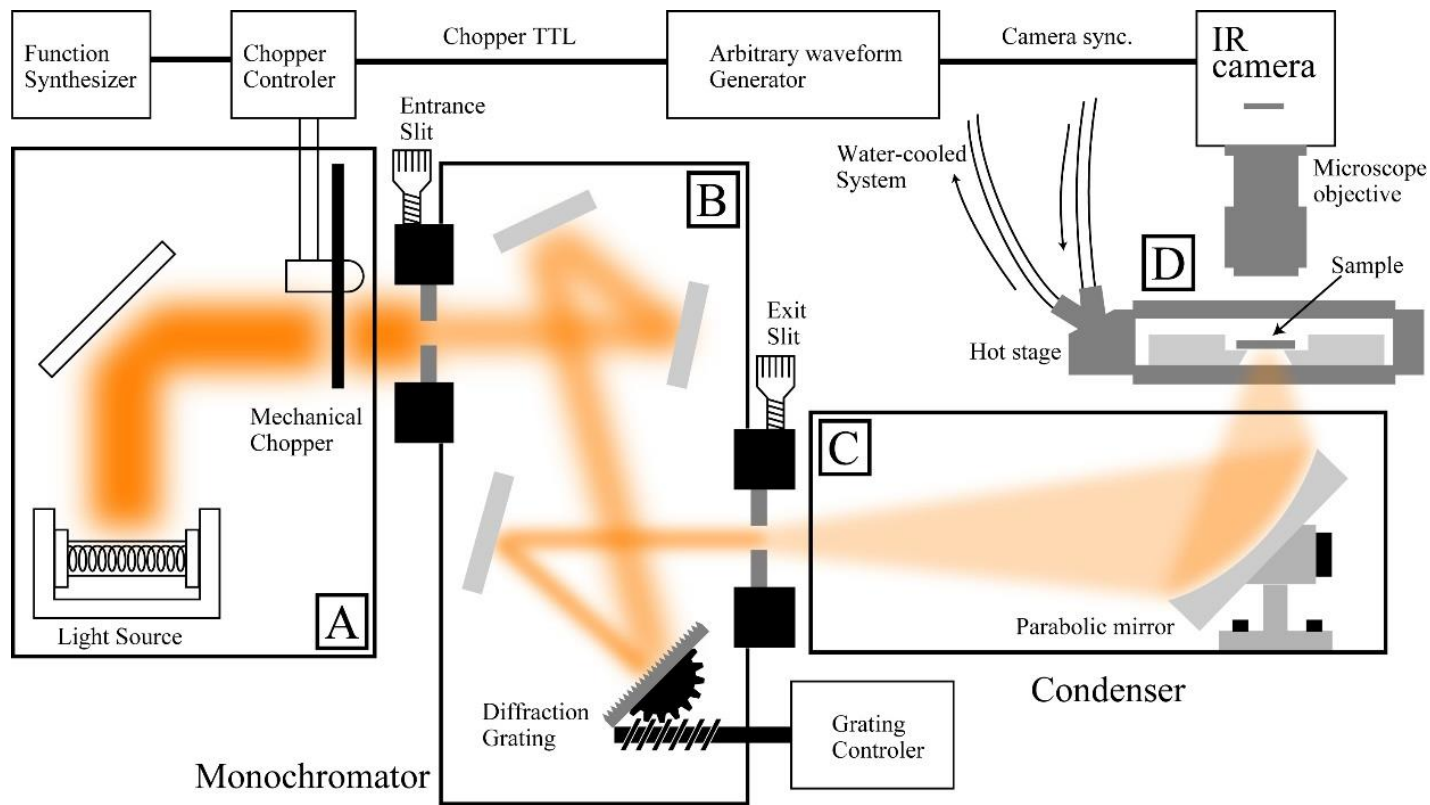

Fig. 1. (A) Ceramic heater (light source), (B) grating monochromator to select the IR wavelength (CT-10, JASCO Corporation), (C) condenser module with a parabolic mirror, (D) thin hot stage (S84, ST Japan) acting also as a sample holder place in a XYZ stage and the IR camera (Phoenix, Indigo).: Measuring setup for high frequency dynamic simultaneous microscopic measurements of thermal and spectroscopic image in mid Infrared wavelength.

\subsection{Chemical substances}

With the developed system in Fig. 1, the typical $n$-alkane, $n$-Tetracosane $\left(\mathrm{C}_{24} \mathrm{H}_{50}\right)$ (Tokyo Chemical Industry, Co. Ltd.) was chosen as an organic phase change material. The phase transition temperatures of $n$-tetracosane determined by temperature wave method $[17,18]$ are $50.2^{\circ} \mathrm{C}$ (liquid state to hexagonal crystal system) and $44.7^{\circ} \mathrm{C}$ (hexagonal to monoclinic crystal system). On cooling scan, the sample was kept at the $60^{\circ} \mathrm{C}$ in the liquid state for 5 minutes to eliminate the thermal history of the sample. The cooling rate of the heat stage is $4{ }^{\circ} \mathrm{C} / \mathrm{min}$. 


\subsection{Processing}

The raw data taken in this apparatus has $128 \times 128$ pixels images including the thermographic images ( $T$ image) and the spectroscopic + thermographic images ( $/$ image). The spectroscopic images ( $S$ image) can be obtained by subtracting $T$ image from $/$ image. Here, we have $5 \mathrm{~ms}$ delay between the $T$ image and I image, thus this subtraction is adequate as long as the phenomena is sufficiently slower than $5 \mathrm{~ms}$. There are two possibilities of subtraction between $I$ and $T$ image as follow,

$$
\begin{aligned}
& S(t)=I(t-\Delta t)-T(t) \\
& S(t)^{\prime}=I(t+\Delta t)-T(t)
\end{aligned}
$$

where $\Delta t$ is the delay between two images. These two subtractions can be used as a verification process of the subtraction. The $S$ image variation that only appears in one of the $S(t)$ or $S(t)$ ' should come from the inappropriateness of the subtraction.

For the static state spectrum acquisition, the raw data is also processed in the same way as mentioned eq1, 2 . Maximum speed of grating motion for the wavelength scan is $1200 \mathrm{~nm} / \mathrm{min}$. In this scan rate, $200 \mathrm{~Hz}$ acquisition of image causes $0.1 \mathrm{~nm}$ step acquisition for the spectrum. However, as the grating rotates by the pulse motor with minimum pitch of $1.6 \mathrm{~nm}$, the data acquisition is performed 16 times in one step of wavelength.

\section{Results and Discussions}

\subsection{Spectroscopy of the sample}

The whole range spectrum of $n$-Tetracosane $\left(\mathrm{C}_{24} \mathrm{H}_{50}\right)$ at each phase measured with the high wavelength resolution $10 \mathrm{~nm}$, which is obtained under the condition $0.15 \mathrm{~mm}$ slit opening width, is shown in Fig. 2(a). The scan range is from $2.76 \mu \mathrm{m}$ to $5.24 \mu \mathrm{m}$. This measurement was performed under the isothermal condition of $26.4^{\circ} \mathrm{C}($ Solid; monoclinic), $45.0^{\circ} \mathrm{C}$ (Rotator; hexagonal), and $57.9^{\circ} \mathrm{C}$ (Liquid).
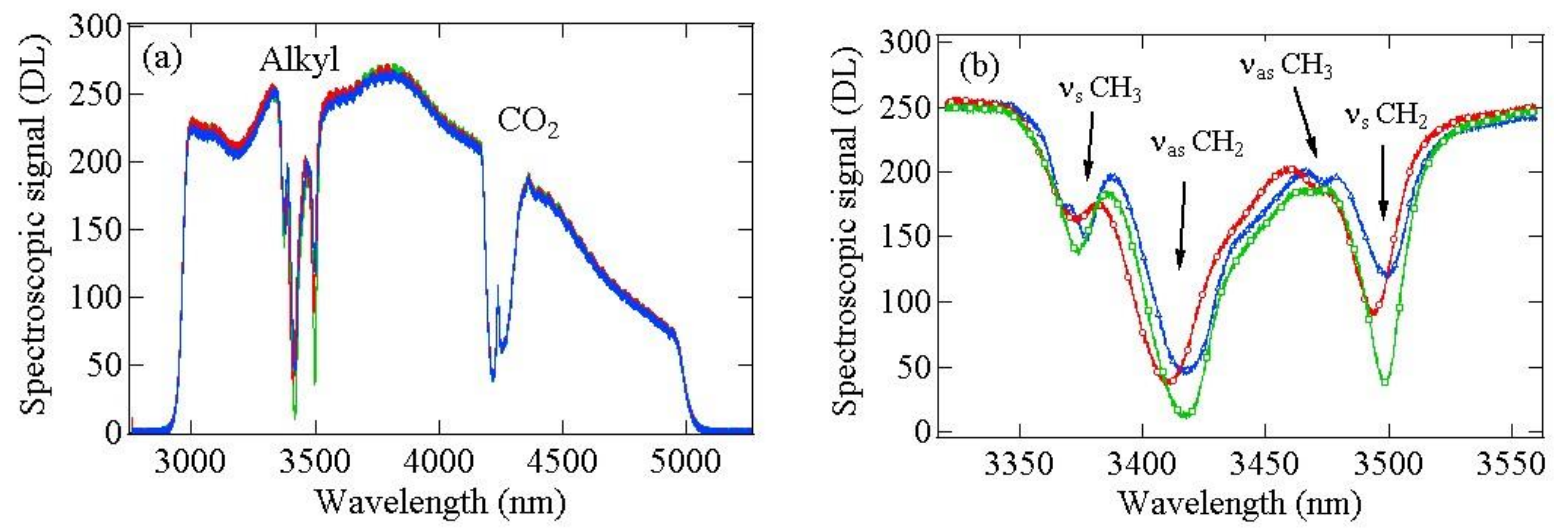

Fig. 2. IR transmission spectrum of $n$-Tetracosane $\left(\mathrm{C}_{24} \mathrm{H}_{50}\right)$ at the liquid phase (red-circle), rotator phase (green-square), and solid phase (blue-triangle). (a) The overall spectrum in the sensitive region of the camera (3-5 $\mu \mathrm{m})$. (b) Zoom on the spectral region of interest concerning the alkyl bands (3.33-3.5 $\mu \mathrm{m})$.

In the alkyl band, we can see small difference of peak positions between three different phases (Figure 2(b)) and several alkyl vibrational modes were distinguished. The absorptions of $\mathrm{CH}_{2}$ and $\mathrm{CH}_{3}$ vibration bands are well characterized. The spectral assignment of the alkyl band is shown in Fig. 2 (b). The four peaks that correspond to the asymmetric and symmetric stretching of $\mathrm{CH}_{2}$ and $\mathrm{CH}_{3}$ were observed. Note that the spectral resolution has been improved compared to the previous reported spectral data for $n$-Eicosane (C20), where these peaks were overlapped [13]. The $200 \mathrm{~Hz}$ modulation of the irradiation light was much faster than the regulation of the heating system; hence the dynamic wavelength scan at $200 \mathrm{~Hz}$ enables us to obtain a stable baseline at each temperature. With this process of baseline subtraction, a small absorption shift can be observed especially at the high temperature. Here, this system has a big advantage even in the static state spectrum measurement, because the spectra at any temperatures are free from the base line variation that comes from the temperature instability of the system such as the regulation of the hot stage. In this dynamic measurement of the spectroscopic and thermographic imaging, the dynamic change of a spectroscopic image at a chosen specific absorption wavelength and the thermal scene image are simultaneously acquired. 


\subsection{Simultaneous Spectroscopic and thermal images}

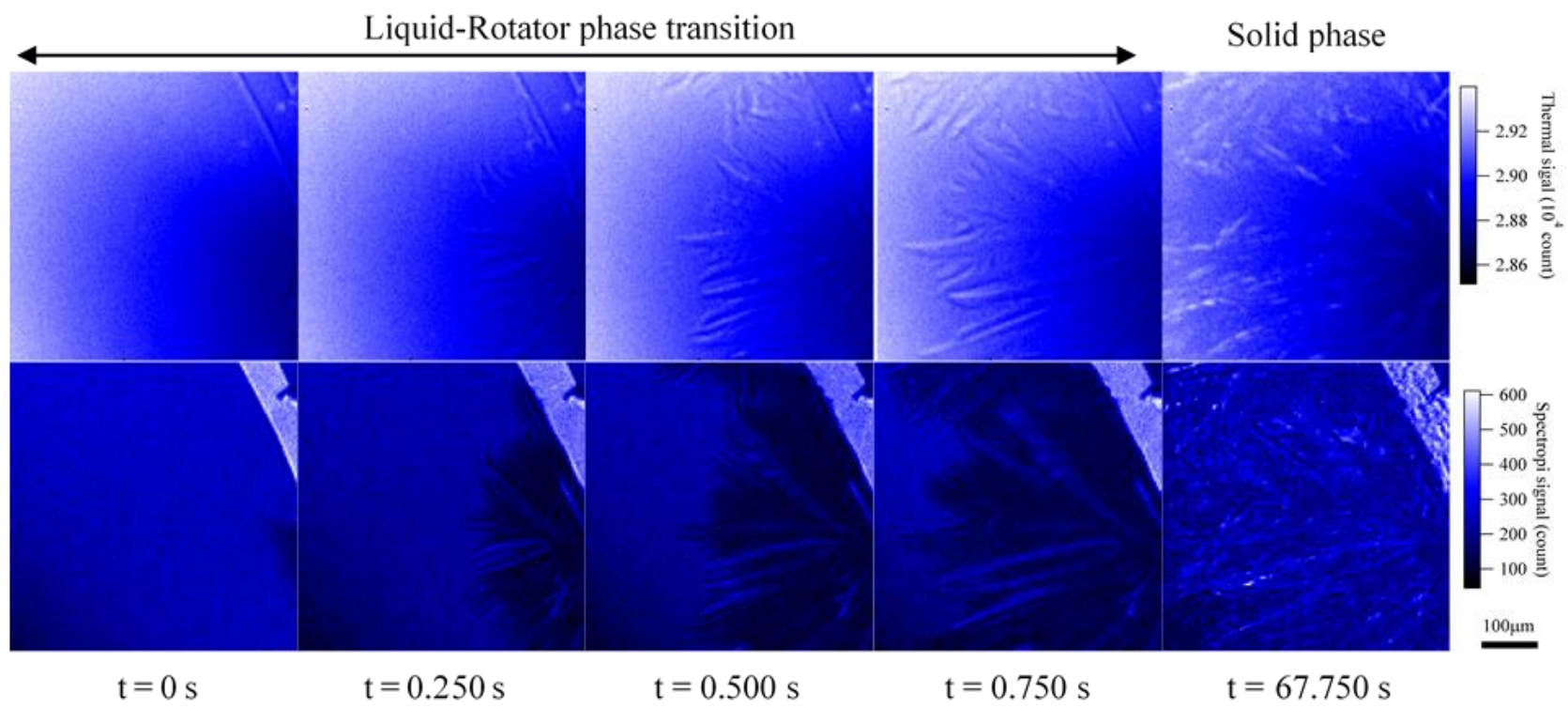

Fig. 3. The $3.47 \mu \mathrm{m}$ IR spectroscopic (lower row) and the thermographic (upper row) images of the rotator (hexagonal) phase evolution in the liquid phase $(t=0 \mathrm{~s}-0.750 \mathrm{~s})$ and the solid phase $(t=67.750 \mathrm{~s})$ of $n$-Tetracosane $\left(C_{24} H_{50}\right)$.

Figure 3 shows the morphological change of the sample during the phase transition. The liquid to solid phase (hexagonal crystal) transition was observed as a lamella growth in the homogeneous liquid phase. In the thermographic image, the only edge of lamella has high signal count, during the evolution of the crystal structure. Then the bright edge fade and diffuse after phase transition due to the cooling in thermal image, while the structure appeared in the spectrum image was still remain after phase transition. For example, the place where the lamella edge is passing through at $0.25 \mathrm{~s}$ is already cooled down at $0.75 \mathrm{~s}$, thus the edge shape of the lamella disappears in the thermal image. They simply indicate the characteristics of both images: the spectrum image is only sensitive to the chemical structure. On the other hand, the rotator (hexagonal crystal system) to solid (monoclinic crystal system) phase transition was observed as a sudden transmittance and emissivity change. The image became bright and the structure became rough texture in the spectrum image.

\subsection{Dynamic phase change analysis}

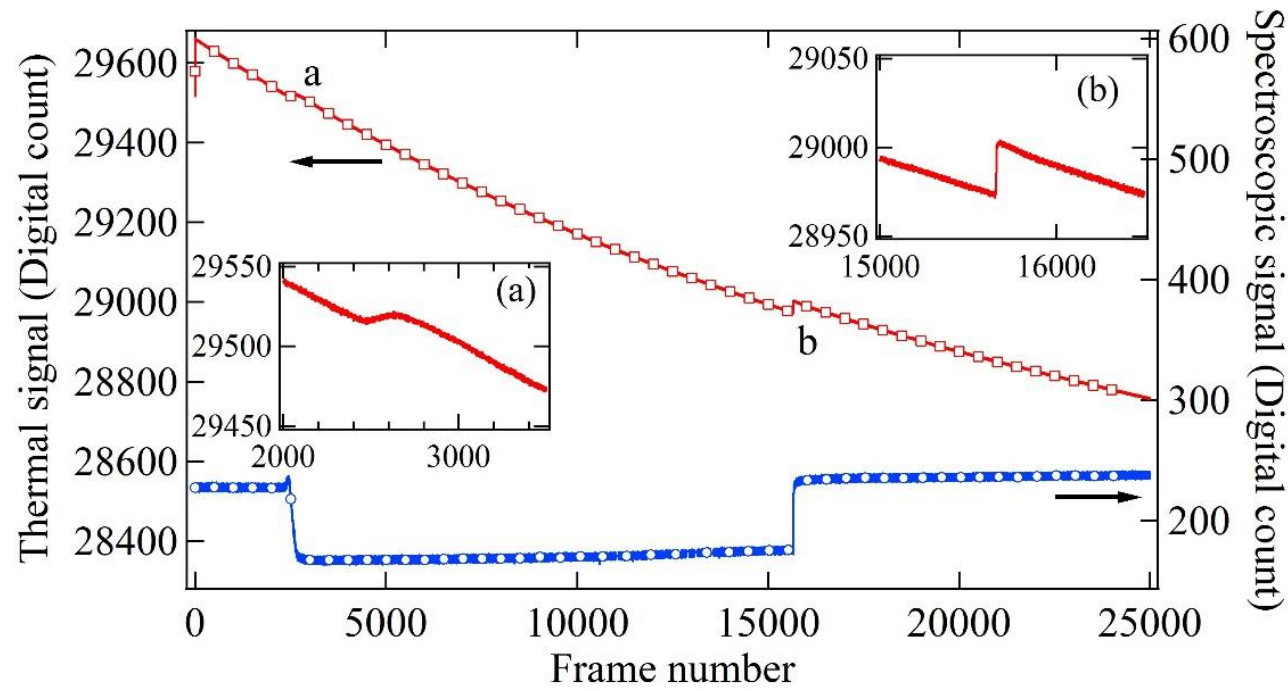

Fig. 4. The thermal (T), thermal $+3.5 \mu \mathrm{m}$ spectroscopic (I), spectroscopic (S) signals taken alternatively at 200 $\mathrm{Hz}$ during the liquid-rotator(hexagonal)-solid(monoclinic) phase transitions of $n$-Tetracosan $\left(\mathrm{C}_{24} \mathrm{H}_{50}\right)$. Signal is averaged 
within $128 \times 128$ pixels area. Upper line (red-circle) corresponds to T signal for left axis and the lower line corresponds to $S$ alone signal for right axis (blue-triangle). The fitted line for the temperature calibration of each phases are also depicted (solid line).

In Figure 4 the intensity (digital counts) of spectroscopic and thermal signals in the dynamic scans are plotted versus frame numbers in the cooling scan. The plotted signals are the spatial averaged-intensities from the area of $128 \times 128$ pixels. The $I$ and $T$ images obtained in the measurement were generated in accordance with the process which has already developed [10]. The horizontal axis of Fig. 4 is the frame number. Frame number 0 is the highest temperature, and 20000 is the lowest when we are monitoring the cooling process.

The spectroscopic signal shows step like change at the phase change while the thermographic signal decrease continuously during the measurement. The reason of these tendencies of two signals comes from the fact that the spectroscopic signal is sensitive to the chemical or phase change, and the thermographic signal is affected by the both temperature and emissivity change of the sample. The spectroscopic signal difference between three phases comes from the alkyl band sift observed in the static state spectrum that is shown in the Fig.2 (b).

During the phase transition, the calibration becomes complex because the multiple phases existed in the field of view. Usually, switching of the calibration curve is performed at one point. It is the assumption that the phase transition occurs within a frame so there are no considerations on the gradual growth of the next phase. In this study, we can measure simultaneously thermographic and spectroscopic images, thus from the spectroscopic signal, the concentration change of the both phases in the view field during the phase transition can be precisely determined. With this information of the concentration variation in the phase transition, it is possible to change our calibration curve gradually between two phases. The temperature on the sample in the view field of our measurement was calculated by this method. The temperature estimation in the two phases co-existing region is more adequate than previous method with the assumption of a step like phase transition [10].

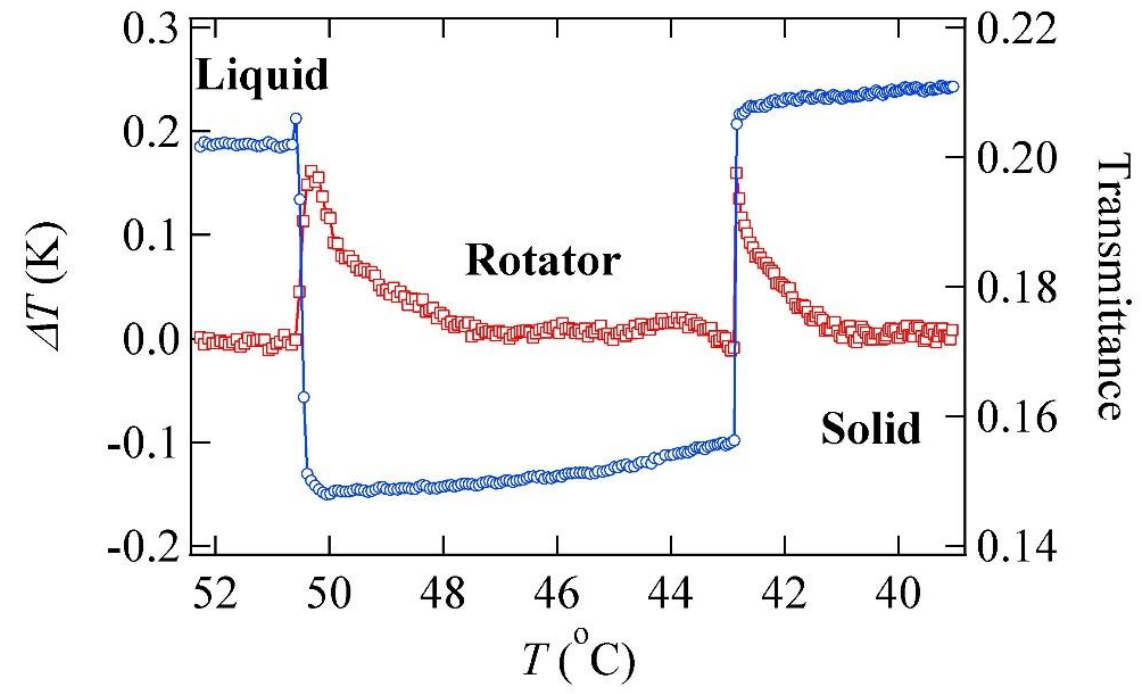

Fig. 5. Temperature difference from the baseline temperature during the cooling scan in the field of view (redtriangle) and $3.5 \mu \mathrm{m}$ transmittance (blue-square), plotted vs. the baseline temperature.

Figure 5 shows the calibrated $T$ signal and $S$ signal. The left axis is temperature difference between the baseline temperature measure by a thermocouple embedded in the hot stage and the calibrated sample temperature from the $T$ signal. The right axis is the $3.5 \mu \mathrm{m}$ transmittance. In the temperature region, which is far from the phase transition, the temperature difference is close to 0 , because the sample and the thermocouple are equally cooled down. Moreover, the spectroscopic signal remains constant in the individual phase, because the sample structure was constant from the vibrational spectroscopic point of view. Twice phase transitions are detected as the two-steps changes in the spectroscopic signal due to the wave-number shift in the spectrum and the temperature increases twice in the thermographic signal because of the exothermic latent heat of $1^{\text {st }}$ order phase transition between solid (monoclinic), rotator(hexagonal), and liquid phases.

\section{Conclusion}

The simultaneous imaging system of microscale IR thermography and spectroscopy was developed, and the possibility of applying it to the observation of dynamic phenomena was demonstrated in this paper. Typical phase change material of $\mathrm{C}_{24} \mathrm{H}_{50}$ ( $n$-Tetracosane) was used as the sample of this dynamic measurement. The measurement was performed at $200 \mathrm{~Hz}$ and this frequency enables to measure the dynamic change of the IR vibrational spectrum and 


\subsection{1/qirt.2016.108}

the thermal images of $n$-alkane at microscale spatial resolutions during phase transitions. In both signals, phase transitions of liquid to rotator (hexagonal) phase, and from rotator (hexagonal) to solid (monoclinic) phase were detected. The thermographic signal contains the exothermic signal and emissivity change in the $1^{\text {st }}$ order phase transition on cooling. The heat generated at the front of phase transition propagates to the surroundings and this diffusion makes the boundary less sharp in the thermographic image. On the other hand, the spectroscopic signal was independent on temperature, thus signal can detect phase transition sharply. With these two signals, the thermographic signal can be converted into temperature and the absolute temperature increase of the sample with exothermic heat during the phase transition was obtained.

The simultaneous imaging system with chemical and thermal information reported in this paper can be combined with many analytical processing. First, a specified image processing algorithms are to be developed to quantitatively track the phase change process that occurs at the transient state of the co-existing phase. Additionally, several thermal models and inverse methods will be proposed for the estimation of the heat release in order to achieve the enthalpy during these dynamics process, also to retrieve the kinetics and the related thermo-physical parameters to be described in such processes.

As the transmitted spectrum signal can be applied to the temperature calibration of the thermal signal, the common problem of the complex thermographic signal is to be partially solved. In other word, the temperature measurement with this method is the concentration independent. The method is effective in the temperature measurement of a complex system such as the concentration distribution field. More generally our technique should be helpful in the characterization of dynamic systems related to the simultaneous variations of both temperature and concentration. In this kind of system, mass and energy phenomena are to be coupled, such as polymer synthesis, reaction diffusion processes, etc. This work shows that there is no obstacle to study ultrafast systems with high space and time resolution by the proposed technique. The high frequency acquisition and the concentration independent temperature measurement make it possible to measure the multidimensional temperature in the dynamic phenomenon in progress.

\section{ACKNOWLEDGEMENTS}

J.M. acknowledges the support of JSPS KAKENHI Grant No. 16 K06768 and 25420752 . Special thanks to Arts et Metiers Paris Tech for the opportunity of research stay of J.M.

\section{REFERENCES}

[1] Y. Hikima, J. Morikawa, T. Hashimoto, "H Wavenumber Dependence of FT-IR Image of Molecular Orientation in Banded Spherulites of Poly(3-hydroxybutyrate) and Poly(I-lactic acid)". Macromolecules, 2013, 46, 1582-1590.

[2] Y. Hikima, J. Morikawa, T. Hashimoto, "FT-IR Image Processing Algorithms for In-Plane Orientation Function and Azimuth Angle of Uniaxially Drawn Polyethylene Composite Film". Macromolecules, 2011, 44, 3950-3957.

[3] Y. Hikima, J. Morikawa, T. Hashimoto, "Imaging of Two-Dimensional Distribution of Molecular Orientation in Poly(ethylene oxide) Spherulite Using IR Spectrum and Birefringence". Macromolecules, 2012, 45, 8356-8362.

[4] S. G. Kazarian, K. L. A. Chan, "FTIR Imaging of Polymeric Materials under High-Pressure Carbon Dioxide". Macromolecules, 2004, 37, 579-584.

[5] S. G. Kazarian, K. L. A. Chan, "'Chemical Photography" of Drug Release”. Macromolecules, 2003, 36, 98669872.

[6] T. Hashimoto, J. Morikawa, "Two-Dimensional Microscale Thermal Analysis of Freezing of Onionskin Cells by High-Speed Infrared Focal Plane Arrays". Jpn. J. Appl. Phys., 2003, 42, L706.

[7] C. Pradere, J. Morikawa, J. Toutain, J.-C. Batsale, E. Hayakawa, T. Hashimoto, "Microscale thermography of freezing biological cells in view of cryopreservation". Quant. InfraRed Thermogr. J., 2009, 6, 37-61.

[8] J. Morikawa, E. Hayakawa, T. Hashimoto, R. Buividas, S. Juodkazis, "Thermal imaging of a heat transport in regions structured by femtosecond laser". Opt. Exp., 2011, 19, 20542-20550.

[9] J. Morikawa, E. Hayakawa, T. Hashimoto, "Micro-Scale Thermal Imaging of Organic and Polymeric Materials with Cooled and Uncooled Infrared Cameras". Advances in Optical Technologies 2012, 2012, 484650.

[10] M. Romano, C. Pradere, F. Sarrazin, J. Toutain, J.C. Batsale, "Enthalpy, kinetics and mixing characterization in droplet-flow millifluidic device by infrared thermography". Chemical Engineering Journal, 2015, 273, 325-332.

[11] C. Sun, D. Xue, "In Situ IR Spectral Observation of NH4H2PO4 Crystallization: Structural Identification of Nucleation and Crystal Growth". J, Phys. Chem. C, 2013, 117, 19146-19153.

[12] A.J. Lopes Jesus, Sandra C.C. Nunes, M. Ramos Silv, A. Matos Beja, J.S.Redinha, "Erythritol: Crystal growth from the melt". International Journal of Pharmaceutics, 2010, 388 129-135.

[13] A. Gupper, K. L. A. Chan, S. G. Kazarian, "FT-IR Imaging of Solvent-Induced Crystallization in Polymers". Macromolecules, 2004, 37, 6498-6503.

[14] M. Romano, C. Ndiaye, A. Duphil, A. Sommier, J. Morikawa, J. Mascetti, J.C. Batsale, L. Servant, C. Pradere, "Fast infrared imaging spectroscopy technique (FIIST)". Infrared Physics \& Technology, 2015, 68, 152-158.

[15] M. Romano, M. Ryu, J. Morikawa, J.C. Batsale, C. Pradere, "Simultaneous microscopic measurements of thermal and spectroscopic fields of a phase change material”. Infrared Physics \& Technology, 2016, 76, 65-71. 
[16] J. Morikawa, T. Hshimoto, E. Hayakawa, T. Eto, " Two-dimensional thermal analysis of organic materials by micro-scale thermography", QIRT 2008 Conference, Krakov, Poland, 2-5 July, 2008, pp. 397 - 401.

[17] N. Miyamoto, J. Morikawa, T. Hashimoto, "Thermal diffusivity of binary mixtures of $n$-tricosane and $n$ tetracosane by Fourier transform temperature wave analysis", Thermochim. Acta, 2015, 431, 62-67

[18] N. Miyamoto, J. Morikawa, T. Hashimoto, "Phase transition of organic molecular crystal observed by thermal diffusivity”, Netsu Sokutei, 2003, 30, 98-105. 